\title{
Structural and non-structural proteins of porcine epidemic diarrhea virus against congenital immunity of host cells
}

\author{
Peng Yuan, Zhou Yang, Yang Yang, Kai Wang, Wei Dong, Han Song, Li Wang and Zhen Hui Song* \\ Department of Veterinary Medicine, Southwest University, Rongchang Campus, Chongqing 402460, People’s Republic of China
}

\begin{abstract}
Porcine epidemic diarrhea virus (PEDV) is a central intestinal pathogen, causing porcine epidemic diarrhea in piglets, this is one of the most vital reasons why plenty of countries have been caught sight of substantial economic losses in animal husbandry. About innate immune response, to some extent, Toll-like receptors(TLRs), transcription and activation of interferon(IFN) resulted a few influences on anti-virus immune. The latter was served as a critical role in the defensive shields against the viral infection. The host could be efficaciously infected by PEDV through the obstacle. There are series of sophisticated strategies to resist virus for host cells. Currently It has been spotted that excellent improvement took place in some studies about the host innate immunity regulated by PEDV. To escape from host-innate immune response PEDV adopts two approaches, originally PEDV encodes viral proteins as interferon antagonists, and its pathogen-associated molecular patterns (PAMPs) would be hided then remain undercover. Non-structural proteins and some proteases of PEDV have been posed some impact on the process. This review illustrates the current grasp on innate immune response of host cells to anti-PEDV covering major proteins between the structural and the non-structural of PEDV to analyze several critical mechanisms on cellular entry of porcine epidemic diarrhea virus, further providing important theoretical grounds for exploring infectious immunity and pathogenic mechanisms of PEDV and supplying the main practical support for its clinical vaccines or medicine research.
\end{abstract}

\section{Introduction}

Porcine epidemic diarrhea virus (PEDV) which can affect swine to infect the epithelial cells lining the small intestine of pigs is a class I of coronavirus [1]. The disease has analogous clinical symptoms compared with transmissible gastroenteritis(TGE), it might be able to bring about diarrhea to engender high mortality rates in suckling piglets, the light of this attack happens without piglets under 4-5 weeks of ages could be primarily used to distinguish PED and TGE [2], even other diseases caused by the intestinal pathogens among most cases. Additionally, wild boars also can be infected by PEDV. Acute watery diarrhea, dehydration and vomiting have been regarded as the chief features for the disease. As for the aspect of economies, PED has been caused vast wastage in different countries. The first confirmed PED which happened on swine was detected from feeder pigs and fattening pigs of Belgium and England in the early 1970s, PEDV was checked as the primary etiological factor roughly 7 years later [3]. From then, in 1982 PEDV has been discovered in some Asian countries within China and Korea leading to high mortality in suckling piglets, sweeping across the much of Asia and bringing about big economic losses on the Asian swine industry since 2010 [4-7]. Afterwards the United states also had been found to be accompanied with PEDV [8].

The innate immune system is always regarded as the first line of defense to protect the host from virus intrusion 9]. Among these influential factors, transcription and activation of type I IFNs (IFN$\alpha / \beta)$ make an extent contribution to stand up to virus. Early reports disclosed that PEDV would inhibit the antiviral activity of IFN through produced defense mechanisms based on the functions of its structural proteins, nonstructural proteins and proteases. Infected vero cells and alveolar macrophages by PEDV was found that production of IFN- $\alpha / \beta$ from the host reduced brightly $[10,11]$. Furthermore, PEDV will decrease IFN- $\alpha / \beta$ activities caused via transfection with doublestranded RNA (dsRNA) of viruses [12]. As for PEDV, there are some pivotal proteins participating in a series of sophisticated anti-viral strategies, not only comprising the structural proteins ( $\mathrm{N}$ protein), but also the nonstructural ones (nsp1, nsp5 and PLP2). In this process, Nucleocapsid protein papain-like proteases of human coronaviruses, SARS-CoV and NL63-CoV could serve as IFN antagonists in the host against viral infection to diminish production of type I interferon synthesized and secreted by eukaryotic cells. During these processes, the viral proteolysis and deubiquitinating activity create a great implication to interfere IFN response against a forcefully replicating RNA virus.

\section{Innate immunity}

As is often the case, some proteins with a specific function are encoded in the anti-infective process of various coronavirus, emerging a set of reaction mechanisms obstructing and escaping host innate immunity little by little, disintegrating the antiviral, surely PEDV has no exception. Multiple defense mechanisms will be appeared to resist outside pathogen invading or monitor and remove abnormal cells in vivo after the body has been infected by viruses, the defense mechanism to protect the host from its invasion can be referred to the innate immune system. Pathogen associated molecular patterns(PAMPs) can be perceived by pattern recognition receptors(PRRs) expressed by cells, which conclude some of conservative molecules in the process of invasion or replication of pathogenic microorganisms. There is

Correspondence to: Xiu-Li Zhang, $\mathrm{PhD}$, Department of Gastroenterology, Chinese PLA General Hospital, Beijing 100853, China, Tel: +86-10-55400305; Fax: +86-10-55400305; E-mail: zhangxl70@126.com

Key words: vascular malformation, gut, coronary artery aneurysm, new syndrome Received: March 11, 2018; Accepted: March 20, 2018; Published: March 26, 2018 
no doubt necessary to produce neutralizing antibody and activate cytotoxic T lymphocyte(CTL) or natural killer cells in the induction of antiviral immune response with the specificity and efficiency, but other host cells still can be provided with immune mechanisms to protect against viral infections. Here the effects of Toll-like receptors in point of antiviral immune or transcription and activation of interferons in the process of host innate immune response about will be demonstrated briefly.

\section{Toll-like receptors(TLRs) in anti-virus immune}

TLRs is a type I transmembrane protein family that can identify different pathogen associated molecular patterns (PAMPs). dsRNA of PEDV recognized by TLR3 is also considered to be the sort of PAMPs. After invasion of virus, produced PAMPs are recognized and combined with extracellular leucine-rich repeat regions. Cytoplasmic regions are responsible for recruitment of the linker molecular proteins containing the TIR domain to stimulate reactions of signaling cascades downstream [13]. After the reorganization of PAMPs by TLRs, TLR regions in the cytoplasm combine with downstream joint molecules to stimulate series of signal transductions to activate transcription factors such as NF- $\kappa$ B, AP-1 and IRF-3, which can regulate production of pro-inflammatory cytokines and chemokines to make the pathogens invasion be inhibited.

TLRs distribute widely in cells can identify the bacterial pathogenimmediate innate immune response and respond antiviral immune response after hosts stimulated by virus. TLR 2 can identify extensive PAMPs concluding bacteria, fungi, parasites and viral derivatives,
TLR4 is a critical receptor for the identification of bacterial lipopolysaccharide (LPS) [14]. Endosome TLRs concluding TLR3, TLR7, TLR8 and TLR9, can be activated after the detection of different types of viral RNA (Figure 1). Expressions of these TLRs will increase the production of type I IFNs and other antiviral cytokines. TLR3 distributes more extensive but mainly express in conventional DCs cells [15]. Overexpression of human TLR3 in 293 cells for dsRNA nonresponsive cell line can enhance the activation of dsRNA-mediated NF- $\mathrm{BB}$. Relative study shows that TLR7 of mice may recognize another compound [16], loxoribine which plays a role of antiviral and antitumor activity $[17,18]$. Thus, TLR7 and TLR8 recognize viral guanine or guanylate-rich ssRNA, such as Human Immunodeficiency Virus(HIV), Vesicular Stomatitis Virus(VSV) and Influenza virus(IV) $[11,16,19]$. TLR9 plays a part through the identification of chromatin structure in the pathogenesis of several autoimmune diseases.

\section{Transcription and activation of interferons}

The host was infected by pathogenic microorganism and monitor pathogen-associated molecular pattern(PAMP) by its wide-distributed pattern recognition receptors(PRRs) in the host, PRRs were activated to trigger a series of phosphorylation or ubiquitin-mediated signaling cascade by recruiting downstream adaptor protein, finally the expression of interferon and pro-inflammatory cytokines was induced by nuclear factor $\kappa \mathrm{B}(\mathrm{NF}-\kappa \mathrm{B})$ and interferon regulatory factors(IRFs) [13].

Interferon (IFN) as well as cellular antiviral response induced by IFN are the foremost defense mechanisms to avoid viral infection. IFN is a type of cytokine of the low relative molecular mass, which

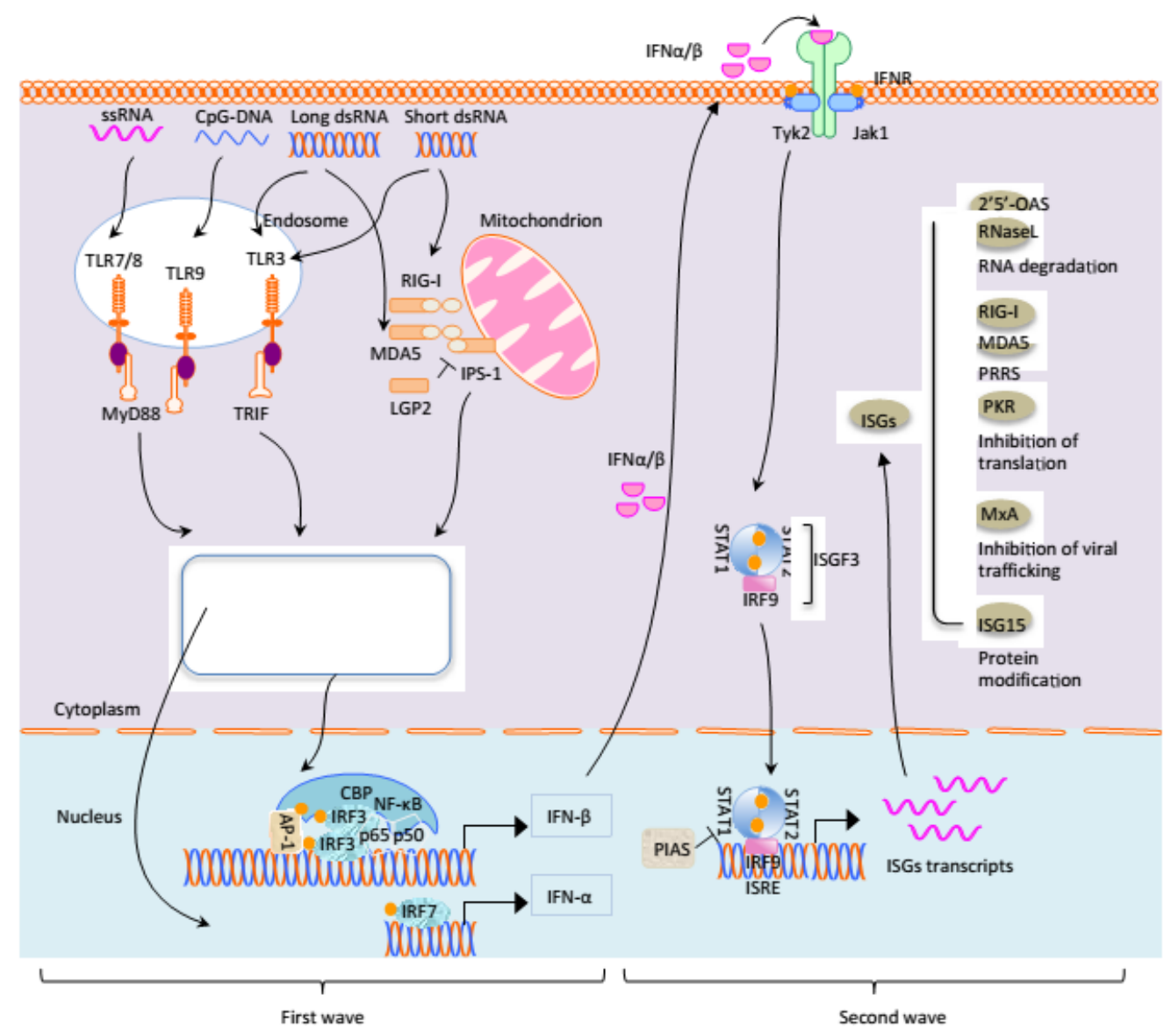

Figure 1. Signal pathways for IFN-I production and relative gene expressions. (Originally posted by Sun Y et al. [7]). 
displays a broad spectrum of biology activities, such as the function of broad-spectrum antivirus, antitumor and immunoregulation. Porcine interferon can be segmented into type I and type II. IFN $\alpha, \beta$ and $\gamma$ are well-known at the moment. IFN- $\alpha$ and IFN- $\beta$ are parts of type I IFN, has a better effect of inhibiting virus replication and antitumor. Type II IFN only has IFN- $\gamma$, primarily involved in immune regulation and inflammatory response.

PEDV could encode a series of proteins to deter transcription and activation of type I IFN during its replication, there are some antagonists perform a function. In the case of virus-negative, type I IFN will come into being from the stimulation in all nucleated cells after the production of IFN, but type II IFN only can be secreted by limited cells, such as natural killer cells, activated T lymphocytes and macrophages [20]. IFN can arouse NK cells and dendritic cells to make adaptive immune system initiate. Expression of interferons and cytokines will be adjusted by interferons and cytokines and activated by germline genesencoded pattern recognition receptors(PRRs). PAMPs of pathogens are recognized by PRRs and the information will be delivered to the downstream signaling molecules, most of RNA virus can be perceived by PRRs (intracellular TLR3, TLR7 and RIG-I or MDA5) to induce generation of type I IFN. The dsRNA of virus replication products can be identified by TLR3 and RIG-I, then the signal should be passed to downstream TANK-binding kinase 1 (TBK1) and inhibitive $\kappa B$ kinase $\varepsilon(\mathrm{IKK} \varepsilon)$ of signaling molecules through its actions on different adapter proteins [21-23]. Activated TBK1/IKKe shall result in activization and phosphorylation of IRF-3. Nuclear translocation will take place in phosphorylated IRF-3 and combine with DNA elements to activate the transcription of IFN $\alpha$ and IFN $\beta(12,31)$. IRF- 3 and IRF-7 play a critical role in the induction and production of IFNa/ $\beta$.

The dsRNA produced in the process of virus replication, a kind of PAMP, which can trigger PRRs to induce expression of IFN $\alpha / \beta$. Tolllike receptors and RIG-I receptor (two PRRs) are mainly responsible for identifying viral dsRNA in the host, expression of IFN- $\beta$ is respectively mediated by TLR3/TRIF pathway and RIG-I/MDA5/IPS1 pathway. The TLR3/TRIF are the primary pathway which can be activated in identification of dsRNA inside cells, but the RIG-I/MDA5/ IPS-1 always can be activated in cytoplasm. IFN does not directly play an antiviral effect after production but combine with its own or specific receptors in contiguous cytomembrane, then expression of multiple effectors molecules can be aroused through the downstream JAK/STAT pathway, such as IFN-induced genes(ISGs) and expression of transcription factor, finally antiviral action will be accomplished. Hence, biological activity does not exist in interferon itself.

Figure 1: During infection, IFN- $\alpha / \beta$ is regulated transcriptionally after recognition of pathogen elements by host pattern recognition receptors. Firstly, in intracellular endosome, viral dsRNA, ssRNA, CpG DNA can separately activate TLR3, TLR7/8 and TLR9, causing dimerization of receptors, the activation of interferon regulatory factor IRF3/IRF7 through assembly of TRIF, NF- $\mathrm{kB}$ activity via recruitment of MyD88 and activation of AP-1 after the initiation of signaling cascades, RIG-I and MDA5 of RIG-I-like helicases can identify viral RNA to initiate IFN- $\beta$ promoter stimulator 1 (IPS- 1 ) adjusting different regulatory factors or kinases by caspase activation and recruitment domains(CARD) of RIG-I and MDA5, so as to promote the activation of IRF3/7 and NF- $\kappa B$ pathways, inducing the transcription of IFN- $\alpha$ and IFN- $\beta$ together with cAMP-response element binding protein(CBP) to produce type I IFN. Subsequently the activation of the JAK/STAT pathway could be resulted from IFN- $\alpha / \beta$ recognition by the IFN receptor (IFNR) on the manner of autocrine and paracrine as soon as
IFN- $\alpha$ and IFN- $\beta$ were secreted into extracellular. STAT1 and STAT2 can be recruited by phosphorylation of the $\mathrm{SH} 2$ region following the phosphorylation of receptor-related JAK1. Grey circle showed the ubiquitination. Yellow circle showed phosphorylation.

IFN $\alpha / \beta$ combined with receptors to phosphorylate JAK and Tyk 2 kinase. JAK and Tyk2 are the activators of STAT1 and STAT2. STAT1 and STAT2 form related dimers of IRF9 after phosphorylation, which can produce interferon stimulated gene 3(ISGF3) complexes. So long as ISGF3 complexes enter nucleus, phosphorylated STAT1 and STAT2 come back to cytoplasm following the dephosphorylation. There are many kinds of ISGs, MxA, OAS-1/RNase L, RIG-I/MDA5, ISG15 and PKR have been mostly researched, which are all antiviral effector molecules.

\section{PEDV in the escape from type I interferons immune response}

Despite of huge antiviral activity of interferon system, PEDV infection also cannot be entirely controlled. Type I IFN (IFN $\alpha / \beta)$ is the most rapid and effective antiviral response in antiviral innate immune response of host. Relatively speaking, PEDV exhibit a certain extent resistance to type I IFN. In the development of evolution, many viruses included PEDV have formed a refined strategy to antagonize interferon pathway to infect and spread effectively in the host cell [24]. Additionally, various proteins are expressed by PEDV express, using different ways for antagonism or escaping from type I interferonmediated antiviral immune response by hiding its PAMPs-dsRNA.

\section{PEDV $\mathrm{N}$ protein in anti-host innate immune}

Nucleocapsid protein from coronavirus is the most abundant protein in the virus- infected cells. Preceding studies have discovered that composition of type I IFN has been inhibited by PEDV infection. Viral papain-like protease 2 was regarded as the IFN antagonist. But [25] found that Sendai virus-induced IFN- $\beta$ production, the activation of IFN regulatory factor 3 (IRF3) and NF- $\mathrm{kB}$ could be suppressed by PEDV N protein, but IRF3-triggered IFN- $\beta$ activation could not be avoided.

There are four IKKs carrying out a critical role in the regulation of the host defense system, which include the classic IKKs (IKKa and IKK $\beta$ ) and the IKK-related kinases (IKKe and TBK1) [26]. TBK1 plays an indispensable part in the signal pathways of infection by RNA virus and inducting IFN production, regulating anti-infective mechanisms of IFN and cytokines. TBK1 (TANK-binding kinase) as one of the IKK-related kinases is very crucial for the phosphorylation and activation of IRF3/7 that can be associated with the protein dimerization, nuclear translocation, DNA binding and the induction of type I interferon (IFN-I) in response to virus infection [22,27-30]. The inhibition or destroy of TBK1 stability is beneficial to suppress the signal transmission of related pathways like TLR and RLR. Further studies demonstrated that PEDV N protein interact with the targeting TBK1 directly, correlation of TBK1 and IRF3 will be dissolved by this combination, while result in inhibiting the activation of IRF3 and production of type I IFN. It shows that TBK1 might be one of the targeted signaling molecules acted by nucleocapsid protein. And related research demonstrated that TBK1 has been able to bind with PEDV N protein and phosphorylate it, thereby having effect on pathogenicity of PEDV to be worthy of discovering.

\section{PEDV nspland nsp5 in anti-innate immune response}

As an antagonist of IFN, 3C or 3C-like proteases of most virus could cleave the signal molecules of type I IFN to suppress natural 
immune response, e.g. NEMO, who is the NF- $\kappa \mathrm{B}$ essential modulator as an critical cohesion adaptor can receive signals from recruitment of mitochondrial antiviral protein like MAVs by RLRs(retinoic acid inducible gene I-like receptors).NEMO regulating fundamentally the IKK compounds is one of the most important critical adaptors for IFN synthesis and also required for NF- $\kappa \mathrm{B}$ activation. PEDV Nsp5 whose cysteine protease activity facilitate proteolysis of NEMO is a kind of IFN antagonist encoding 3C-like protease at glutamine 231 (Q231) [31], causing the radical influence on the strategic interactions between virus and host, which is decisive factor to the mechanism of PEDV infection.

The other nonstructural protein of PEDV is nsp1, also can be the IFN antagonists of related virus to detect how to squelch type I IFN response by dependent on proteasome pathway and inhibit the host IFN gene synthesis $[32,33]$, especially IFN- $\beta$ production always become the prime targeted object inhibited after cells infected by PEDV as soon as cloning and expressing the entire viral gene sequences including the structural and nonstructural proteins. Expect for inhibition of IFN- $\beta$, like other PEDV nonstructural proteins (nsps) nsp3, nsp7 and nsp14 16, the nsp1 also have been discovered that inhibitory effects on IRF3 promoter activities. It can interrupt stiffened composition of interferon regulatory factors 3 (IRF3) and CREB (cAMP responsive element binding)-binding protein (CBP) through degradation of CBP, but not hold back the IRF3 phosphorylation or nuclear translocation which is formed in the process of demasking nuclear localization signal after homodimerization by phosphorylated IRF3 upon TBK1 activation [34], which could bind with PEDV N protein, playing an important role on pathogenicity of PEDV.

\section{dsRNA and PLP2 of PEDV were concealed to evade innate immune response}

Both PAMPs and PLP2 become the most important approaches for PEDV to evade innate anti-viral immune response. There are some stable molecular structures from the surface of pathogens, which are evolution of highly conserved and shared by a great deal of relevant microorganisms but without in human, such as doublestranded RNA(dsRNA) of viruses and bacterial lipopolysaccharide. Pattern recognition receptors (PRRs are regarded as immunoreceptors expressed by cells of the immune system and spotting pathogenassociated molecular patterns (PAMPs), RLR receptor of which have the inseparable relations with discernment of viruses. RIG-I, MDA5 and LGP2 have been summed up to the three categories of RLR receptors. RIG-I and MDA5 can identify dsRNA from viruses to sense different invasion of virus. And then amount of dsRNA appear from the intracellular when facing to viral infections, to induce production of antiviral IFN-I. As a class of RNA helicase in the cytoplasm, RIG-I receptors are known to combine PAMPs to identify viral dsRNA containing 5'-triphosphate, but not from itself [35], long dsRNA also can be combined by MDA5 specifically [36]. Through these interactions infected cells could trigger activation of RLRs downstream signal molecules to significantly promote the production of IFN-I and relevant inflammatory factors, resulting in antiviral immune response. There is a self-assembled process that $5^{\prime}$-cap structures increasing the stability of mRNA and efficiency of translation on eukaryotic mRNA are formed in transcription after the modification by related enzymes. RLR cannot recognize cap-structures which don't be methylated in the 5'-end of RNAs. Towards RNA modification of coronaviruses, 2'-O-methylation and N7-methylation become the most important parts during lots of mRNA-related processes seem to be helpful for the discrepancy of self and non-self-viral RNA in respond to innate antiviral immunity, methylating 5 ' cap structures of their mRNA [3740]. For example, the lack of 2'-O-MTase activity could be sensitive to IFN-I to bring about the increasing of its expression. Separately 2'-O-methyltransferase and N7-methylation was encoded by nsp16 and nsp14 of coronaviruses to make the methylated cap structures have been projected on viral mRNA to avoid the recognition of RLR through the fading of their RNA.

Coronaviruses, covering porcine epidemic diarrhea virus(PEDV), protease was known as the most critical protein being beneficial to viral RNA synthesis all viral replication cycles. Except for protease activity, the decorated functions of ubiquitination and deubiquitination for relevant protein molecules play an extremely vital role in regulation of immune reaction among innate immune signal pathways. PLP2 encoded by PEDV has been served as an antagonist of type I IFN and is helpful to inhibit IFN-I expression to evade innate immunity because of its deubiquitination DUB activity and antagonistic activity of interferon. According to preliminary studies, human NL63 CoV (HCoV-NL63) [41] was found that its papain-like proteases(PLP) also have been provided with DUB activity and it can reduce the IFN activity account of its antagonistic effects [42]. There was a kind of human coronavirus, called severe acute respiratory syndrome coronavirus (SARS-CoV), it has been discovered that generates about six antagonists of innate immunity, especially the PLP regions exist in (non-structural protein 3) nsp3 of SARS-CoV [43]. As a multifunctional protein, PLP2 encoded by PEDV is a fresh coronaviral DUB whose critical sites of DUB activity is identical to the catalytic active sites of PLP2 based on the construction of protease mutants of PLP2 catalytic active sites using the site-directed mutagenesis, indicating that the ingenious structure of PLP2 is the basis on the function against innate antiviral immune response. But RIG-I and STING can be identified by PLP2 of PEDV to separate the ubiquitinated combinations from the two proteins is how PEDV PLP2 inhibits IFN response.

\section{Conclusion}

As we know, national economy development of a country was covered by various fields of agriculture, commerce, industry to promote coordinated growth of various regions which could be very essential to national construction. However, agricultural economy's development combining livestock appears to be very critical with a relatively high status and role. Including PEDV, most of enteric coronaviruses can cause the spread of the epidemic diarrheal diseases, resulting in local infection even large-scale outbreak in pig population, bringing about big economic losses in pig industry then the development of animal husbandry must be threatened. It is primarily helpful to PEDV infect the host following up the damage of Innate immunity defence, while virus invade the body, innate immune cells will immediately initiate reactions of viral recognition and anti-virus to product molecular with antiviral functions, Toll-like receptors (TLRs) and interferons play the dominant role. During series of defensive functions were destroyed, some structural and non-structural proteins of PEDV have some impact to a certain extent.

This paper suggests that functional attributes of few significant proteins to explain they how to evade specific antiviral cytokines (IFN $\alpha / \beta$ ) and defensive functions of a type I transmembrane protein (Toll-like receptors) to make incursions into the host smoothly. Structural protein (N protein could bind with TBK1 though weaking combinations of IRF3 and TBK1 to inhibit IRF3 phosphorylation due to TBK1, giving rise to miss the signal reception from TLR and RLR to reduce activation of IRF3, expression of $\operatorname{IFN}(\alpha / \beta)$ and ISG3 to abate 
antiviral effect. PEDV nsp5 protein regarded as interferon antagonist can encode 3C-like protease then hydrolyze NEMO which serve as a necessary signal molecule in IFN response to suppress functions of NF$\kappa \mathrm{B}$. nsp3 protein not only can weaken IFN- $\beta$ production in anti-viral process, but also the binding between IRF3 and CREB, not the IRF3 phosphorylation. DUB function and IFN antagonistic activation of PEDV PLP2 are favorable for resist congenital immunity because of its ingenious structural functions. In addition, the unmethylated 5'-capstructures for PEDV mRNA can't be spotted by pattern recognition receptor RLRs to shirk innate immune response. Deeply understanding of how to evade the host defense strategies on antiviral innate immune response by PEDV is beneficial to reveal pathogenesis of PEDV as well as the discovery of new targets for antiviral drugs, thereby laying the foundation for prevention and control of PEDV.

\section{Funding}

This work was supported by the Fundamental Research Funds for the Central Universities (XDJK2017D077, XDJK2017D082).

\section{References}

1. Chen J, Wang C, Shi H, Qiu H, Liu S, et al. (2010) Molecular epidemiology of porcine epidemic diarrhea virus in China. Arch Virol 155: 1471-1476. [Crossref]

2. Pritchard GC, Paton DJ, Wibberley G, Ibata G (1999) Transmissible gastroenteritis and porcine epidemic diarrhoea in Britain. Vet Rec 144: 616-618. [Crossref]

3. Pensaert MB, de Bouck P (1978) A new coronavirus-like particle associated with diarrhea in swine. Arch Virol 58: 243-247. [Crossref]

4. Lund JM, Alexopoulou L, Sato A, Karow M, Adams NC, et al. (2004) Recognition of single-stranded RNA viruses by Toll-like receptor 7. Proc Natl Acad Sci U S A 101: 5598-5603. [Crossref]

5. Puranaveja S, Poolperm $\mathrm{P}$, Lertwatcharasarakul $\mathrm{P}$, Kesdaengsakonwut $\mathrm{S}$, Boonsoongnern A, et al. (2009) Chinese-like strain of porcine epidemic diarrhea virus, Thailand. Emerg Infect Dis 15: 1112-1115. [Crossref]

6. Li W, Li H, Liu Y, Pan Y, Deng F, et al. (2012) New variants of porcine epidemic diarrhea virus, China, 2011. Emerg Infect Dis 18: 1350-1353. [Crossref]

7. Sun RQ, Cai RJ, Chen YQ, Liang PS, Chen DK, et al. (2012) Outbreak of porcine epidemic diarrhea in suckling piglets, China. Emerg Infect Dis 18: 161-163. [Crossref]

8. Stevenson GW, Hoang H, Schwartz KJ, Burrough ER, Sun D, et al. (2013) Emergence of Porcine epidemic diarrhea virus in the United States: clinical signs, lesions, and viral genomic sequences. J Vet Diagn Invest 2: 649-654. [Crossref]

9. O'Neill LA, Bowie AG (2010) Sensing and signaling in antiviral innate immunity. Curr Biol 20: R328-R333. [Crossref]

10. Charley B, Riffault S, Van Reeth K (2006) Porcine innate and adaptative immune responses to influenza and coronavirus infections. Ann N Y Acad Sci 1081: 130-136. [Crossref]

11. Laude H, Reeth KV, Pensaert M (1993) Porcine respiratory coronavirus: molecular features and virus-host interactions. Vet Res 2: 125-150. [Crossref]

12. Miller LC, Laegreid WW, Bono JL, Chitko-McKown CG, Fox JM (2004) Interferon type I response in porcine reproductive and respiratory syndrome virus-infected MARC-145 cells. Arch Virol 149: 2453-2463. [Crossref]

13. Zhanc Y, Zhonu B, Yanu Y (2009) Mechanisms and regulations of TLRs- and RLRsmediated cellular antiviral signaling. Chinese J Cell Biol 31: 453-468.

14. Suresh R, Mosser DM (2013) Pattern recognition receptors in innate immunity, host defense, and immunopathology. Adv Physiol Educ 37: 284-291. [Crossref]

15. Iwasaki A, Medzhitov R (2010) Regulation of adaptive immunity by the innate immune system. Science 327: 291-295. [Crossref]

16. Heil F, Hemmi H, Hochrein H, Ampenberger F, Kirschning C, et al. (2004) Speciesspecific recognition of single-stranded RNA via toll-like receptor 7 and 8. Science 303: 1526-1529. [Crossref]

17. Lee J, Chuang T, Redecke V, She L, Pitha PM, et al. (2003) Molecular basis for the immunostimulatory activity of guanine nucleoside analogs: activation of Toll-like receptor 7. Proc Natl Acad Sci U S A 100: 6646-51. [Crossref]
18. Eil F, Ahmad-Nejad P, Hemmi H, Hochrein H, Ampenberger F, et al. (2003) The Toll-like receptor 7 (TLR7)-specific stimulus loxoribine uncovers a strong relationship within the TLR7,8 and 9 subfamily. Eur J Immunol 33: 2987-2997. [Crossref]

19. Diebold SS, Kaisho T, Hemmi H, Akira S, Reis e Sousa C (2004) Innate antiviral responses by means of TLR7-mediated recognition of single-stranded RNA. Science 303: 1529-1531. [Crossref]

20. Longhi MP, Trumpfheller C, Idoyaga J, Caskey M, Matos I, et al. (2009) Dendritic cells require a systemic type I interferon response to mature and induce CD4+ Th1 immunity with poly IC as adjuvant. $J$ Exp Med 206: 1589-1602. [Crossref]

21. Fitzgerald KA, McWhirter SM, Faia KL, Rowe DC, Latz E, et al. (2003) IKKepsilon and TBK1 are essential components of the IRF3 signaling pathway. Nat Immunol 4 491-496. [Crossref]

22. Sharma S, tenOever BR, Grandvaux N, Zhou GP, Lin R, et al. (2003) Triggering the interferon antiviral response through an IKK-related pathway. Science 300: 1148-1151. [Crossref]

23. Ishii KJ, Kawagoe T, Koyama S, Matsui K, Kumar H, et al. (2008) TANK-binding kinase-1 delineates innate and adaptive immune responses to DNA vaccines. Nature 451: 725-729. [Crossref]

24. Haller O, Weber F (2007) Pathogenic viruses: smart manipulators of the interferon system. Curr Top Microbiol Immunol 316: 315-334. [Crossref]

25. Ding Z, Fang L, Jing H, Zeng S, Wang D, et al. (2014) Porcine epidemic diarrhea virus nucleocapsid protein antagonizes beta interferon production by sequestering the interaction between IRF3 and TBK1. J Virol 88: 8936-8945.

26. Häcker H, Karin M (2006) Regulation and function of IKK and IKK-related kinases. Sci STKE 2006: re13. [Crossref]

27. Lin R, Mamane Y, Hiscott J (2000) Multiple regulatory domains control IRF-7 activity in response to virus infection. J Biol Chem 275: 34320-34327. [Crossref]

28. Lin R, Heylbroeck C, Pitha P, Hiscott J (1998) Virus-dependent phosphorylation of the IRF-3 transcription factor regulates nuclear translocation, transactivation potential, and proteasome-mediated degradation. Mol Cell Biol 18: 2986-2996. [Crossref]

29. Servant M, Grandvaux N, ten Oever B, Duguay, D, Lin R, et al. (2003) Identification of the minimal phosphoacceptor site required for in vivo activation of interferon regulatory factor 3 in response to virus and double-stranded RNA. J Biol Chem 278: 9441-9447. [Crossref]

30. Clément JF, Bibeau-Poirier A, Gravel SP, Grandvaux N, Bonneil E, et al. (2008) Phosphorylation of IRF-3 on Ser 339 generates a hyperactive form of IRF-3 through regulation of dimerization and CBP association. J Virol 82: 3984-3996. [Crossref]

31. Wang D, Fang L, Shi Y, Zhang H, Gao L, et al. (2015) Porcine Epidemic Diarrhea Virus 3C-Like Protease Regulates Its Interferon Antagonism by Cleaving NEMO. $J$ Virol 90: 2090-2101. [Crossref]

32. Tohya Y, Narayanan K, Kamitani W, Huang C, Lokugamage K, et al. (2009) Suppression of host gene expression by nsp 1 proteins of group 2 bat coronaviruses. $J$ Virol 83: 5282-5288. [Crossref]

33. Kamitani W, Huang C, Narayanan K, Lokugamage KG, Makino S (2009) A twopronged strategy to suppress host protein synthesis by SARS coronavirus Nsp1 protein. Nat Struct Mol Biol 16: 1134-1140. [Crossref]

34. Zhang QZ, Shi K, Yoo D (2016) Suppression of type I interferon production by porcine epidemic diarrhea virus and degradation of CREB-binding protein by nsp1. Virology 489: 252-268. [Crossref]

35. Hornung V, Ellegast J, Kim S, Brzózka K, Jung A, et al. (2006) 5'-Triphosphate RNA is the ligand for RIG-I. Science 314: 994-997. [Crossref]

36. Kato H, Takeuchi O, Mikamo-Satoh E, Hirai R, Kawai T, et al. (2008) Lengthdependent recognition of double-stranded ribonucleic acids by retinoic acid-inducible gene-I and melanoma differentiation-associated gene 5. J Exp Med 205: 1601-1610. [Crossref]

37. Bouvet M, Debarnot C, Imbert I, Selisko B, Snijder EJ, et al. (2010) In vitro reconstitution of SARS-coronavirus mRNA cap methylation. PLoS Pathog 6: e1000863. [Crossref]

38. Chen Y, Cai H, Pan J, Xiang N, Tien P, et al. (2009) Functional screen reveals SARS coronavirus nonstructural protein nsp14 as a novel cap N7 methyltransferase. Proc Natl Acad Sci U S A 106: 3484-3489. [Crossref]

39. Decroly E, Imbert I, Coutard B, et al. (2008) Coronavirus nonstructural protein 16 is a cap-0 binding enzyme possessing (nucleoside-2'O)-methyltransferase activity. $J$ Virol 82: 8071. [Crossref] 
Yuan P (2018) Structural and non-structural proteins of porcine epidemic diarrhea virus against congenital immunity of host cells

40. Snijder EJ, Bredenbeek PJ, Dobbe JC, Thiel V, Ziebuhr J, et al. (2003) Unique and conserved features of genome and proteome of SARS-coronavirus, an early split-off from the coronavirus group 2 lineage. J Mol Biol 331: 991-1004. [Crossref]

41. van der Hoek L, Sure K, Ihorst G, Stang A, Pyrc K, et al. (2005) Croup is associated with the novel coronavirus NL63. PLoS Med 2: e240. [Crossref]
42. Chen Z, Wang Y, Ratia K, Mesecar AD, Wilkinson KD, et al. (2007) Proteolytic processing and deubiquitinating activity of papain-like proteases of human coronavirus NL63. J Virol 81: 6007-6018. [Crossref]

43. Devaraj SG, Wang N, Chen Z, Chen Z, Tseng M, et al. (2007) Regulation of IRF3-dependent innate immunity by the papain-like protease domain of the severe acute respiratory syndrome coronavirus. J Biol Chem 28: 32208-32221. [Crossref]

Copyright: @2018 Yuan P. This is an open-access article distributed under the terms of the Creative Commons Attribution License, which permits unrestricted use, distribution, and reproduction in any medium, provided the original author and source are credited. 